\title{
Linx
}

Revue des linguistes de l'université Paris X Nanterre

5 | 1994

La négation

\section{La rhétorique de la négation}

Hildegard Vermeiren

\section{OpenEdition}

\section{Journals}

Édition électronique

URL : http://journals.openedition.org/linx/1230

DOI : 10.4000/linx.1230

ISSN : 2118-9692

\section{Éditeur}

Presses universitaires de Paris Nanterre

\section{Édition imprimée}

Date de publication : 1 juin 1994

Pagination : 331-341

ISSN : 0246-8743

\section{Référence électronique}

Hildegard Vermeiren, "La rhétorique de la négation », Linx [En ligne], 5 | 1994, mis en ligne le 18 juillet 2012, consulté le 19 avril 2019. URL : http://journals.openedition.org/linx/1230 ; DOI : 10.4000/ linx. 1230

Ce document a été généré automatiquement le 19 avril 2019.

Département de Sciences du langage, Université Paris Ouest 


\title{
La rhétorique de la négation
}

\author{
Hildegard Vermeiren
}

1 Notre point de départ est la constatation que la linguistique limite le plus souvent son objet d'étude à des phrases. C'est aussi bien le cas dans la recherche en langue maternelle - quand le chercheur se base sur sa propre compétence - que dans la recherche en langue étrangère, qui, au moyen d'un corpus, préfère se baser sur une compétence empruntée. Ainsi, dans l'étude de la négation en espagnol, nous constatons qu'Ibáñez (1972) et Bosque (1980) se basent sur leur compétence d'hispanophones, alors que l'allemand Voigt (1979) nous affirme de façon explicite la nécessité de se baser sur un corpus.Nous sommes convaincus que de nombreux problèmes linguistiques - notamment sémantiques peuvent être éclairés au niveau de la phrase. Cependant, nous remarquons que dans le cas de la négation on n'a jamais exploré les dimensions sémantiques de cet élément grammatical au-delà du niveau de la phrase. En d'autres mots: on n'a jamais étudié le fonctionnement de la négation à l'intérieur d'un texte, sans considérer ce texte comme un simple « corpus».

Lorsqu'on s'intéresse, justement, aux négations à l'intérieur d'un texte, on est bien obligé - même plus que dans le cas de l'étude de simples phrases - de se baser sur un corpus concret. La suite de notre raisonnement se fera donc en nous basant sur un exemple concret. Nous avons choisi l'article Cuba : una Numancia en el Caribe, que nous avons trouvé par hasard dans le quotidien madrilène El País du 2 juin 1992, et qui a attiré notre attention tout d'abord par son thème. Ce n'est qu'en le lisant et en le relisant, que nous avons constaté le rôle assez exceptionnel de ses négations.

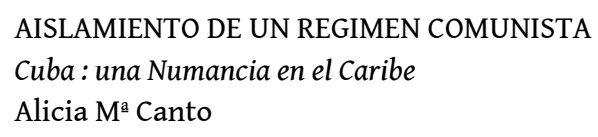

3 La autora compara la situación actual de Cuba con la Numancia destruida por los romanos, y señala que « el cerco (...) secundado tan dócilmente por tantos países, (...) está en vías de reducir por el hambre y la necesidad a la población ». En su opinión, es una ironía que tal acoso se efectúe precisamente en este año del quinto centenario del descubrimiento de América. 
§.1. El historiador Polibio de Megalópolis fue uno de aquellos orgullosos y cultos griegos, vencidos definitivamente en el año 146 a.C. por los (1) incultos, pero poderosos romanos. Griegos que llegaron a Italia, reducidos a la condición jurídica de esclavos, para servir en las casas de Roma. Polibio tuvo suerte, ya que, gracias a sus amplios conocimientos culturales, entabló relación con los Medici de la época, los Escipiones. Andando el tiempo, el griego tuvo ocasión de viajar a Hispania, dentro del séquito de P. Cornelio Escipión, para asisitir al último acto de una tragedia bélica que era ya un escarnio para la dignidad del más fuerte imperio del momento. Lo que aquí vio le pareció digno de ser recordado.

§.2. En efecto, desde hacía 10 largos años, las temidas legiones romanas habían sido (2) incapaces de vencer a la pequeña ciudad celtibérica de Numancia. Habían fracasado ante sus muros, sucesivamente, los generales Q. Pompeyo (años 141 y 140), Popilio Lenas (138) y Hostilio Mancino (137). Había fracasado también el piadoso T. Graco, que, ante la crueldad y el afán de rapiña con que se trataba a los indígenas, tuvo una conversión como dijeron Bloch y Carcopino, volviéndose defensor, sobre todo desde su experiencia numantina en el 137, de los débiles y oprimidos.

6 §.3. En octubre del año 134 a.C. llega a Hispania P. Cornelio Escipión Emiliano, miembro de una laureadísima familia, destructor y salinizador de Cartago, sumando en su ejército 20.000 soldados. Hombre de moral materialista y (3) desnaturalizada, dispuesto a cosechar el éxito que (4) no pudieron alcanzar los citados, sus primeras medidas fueron, por una parte, atacar a los vacceos, impidiéndoles así que pudieran auxiliar a Numancia y, por otra, disponer un fuerte cerco en torno a la pequeña ciudad. Sólo la vecina Lutia, se atrevió a ayudar a Numancia. Fueron los más jóvenes quienes reunieron el valor para aprestarse a la lucha ; mientras, los mismos ancianos de la ciudad avisaban al romano de la alianza. Sobre Lutia cayó de inmediato la represión de Roma : a 400 de sus jóvenes les fueron amputadas las manos.

\section{Rendición (5) sin condiciones}

7 \$.4. Otras tribus fueron disuadidas mediante la presión diplomática o la amenaza de (6) no ser ayudadas en el futuro por Roma... Varios autores además de Polibio, como Apiano o Livio, describen con detalle la disciplina férrea impuesta por Escipión a sus ejércitos y el brutal asedio. Escipión quería una rendición (7) sin condiciones, no paz ni acuerdos $n i$ tributos.

§.5. (8) Sin posibilidad de recibir ayuda alguna, neutralizados sus aliados, con siete campamentos, fosas y empalizadas a su alrededor, cortados los suministros de agua potable, de madera, carnes o creales, la lenta agonía de Numancia fue llevando a la desesperación a su habitantes. Su resistencia, que unánimemente ha sido calificada por el juicio de la historia como heroica, más relevante cuanto mayor era la desproporción entre ella y su adversario, tiene detalles espeluznantes y de todos conocidos. Pero Numancia (9) no se rindió : murieron todos revueltos, hombres, mujeres y niños. Famélicos, calcinados en confuso amasijo con ideales que, desde el materialismo de Escipión, era (10) inútil tratar de comprender o defender: un amasijo compuesto de otra lengua, otros dioses, otros modos de vivir y entender los medios de producción, la propiedad común de la 
tierras, la adorada (11) independencia o la (12) negativa a entregar sus campos y sus ciudades al depredador romano. Para virtudes y defectos preferían, en cualquier caso, los propios.

9 §.6. Las ruinas de Numancia, que cautivaron, entre otros, al arqueólogo alemán Schulten, pueden ser visitadas cerca de Garray, en Soria. Roma se impuso al final, pero (13) nadie ha discutido nunca que lo logró por la fuerza y la brutalidad,no por la razón.

\section{Sacrificio lamentable}

§.7. La memoria de Numancia (14) no se consumió, ni mucho menos. Pero fue un sacrificio lamentable. ¿ Quién defendería hoy que los numantinos (15) no tenían el derecho de ser como querían ser? Libres de elegir su propio futuro, o de (16) no tener ninguno.

11 §.8. Lo siento, pero el caso de Cuba tiene muchas similitudes históricas con el de Numancia. El mismo cerco que, llevado hoy desde la distancia y secundado dócilmente por tantos países, parece aún más cobarde, está en vías de reducir por el hambre a una numerosa población. (17) No parece importar que mueran millones de personas de hambre, o de enfermedad, por la falta de medicinas; o que acaben matándose unos a otros para poder comer, siempre que se consiga doblegar a uno solo; siempre que se consiga que su poderoso vecino de norte pueda sacarse por fin la dolorosa espina de una prolongada derrota, más imaginaria que real.

§.9. Está visto que la historia (18) no enseña casi nada a los hombres. Y si ello es más esperable en pueblos que tradicionalmente (19) desconocen otra historia que la suya propia, que es la única que les interesa en realidad, es (20) imperdonable que España, que fue tantas veces el escenario y el modelo de ese espíritu de resistencia e (21) independencia , que obligó a Roma a guerrear en su suelo durante 200 años, antes de ser dominada, contemple (22) impasible, día a día, cómo allá, tras las fosas y las empalizadas, se reduce por el hambre y la miseria a un país pequeño y debilitado, que cantaba y reía en nuestro mismo idioma y donde la gente lleva nuestros apellidos. Y es, simplemente, una ironía de la histroria que ello se consume, precisamente, en 1992.

13 Alicia María Canto de Gregorio es profesora titular del Departamento de Prehistoria y Arqueología de la Universidad Autónoma de Madrid.

\section{Qu'est-ce que nous voulons dire par « négation »?}

14 Tout d'abord nous voulons remarquer que nous nous occupons uniquement de la négation en espagnol et que des perspectives contrastives ou le point de vue « universel » ne nous intéressent pas ici. Ensuite, il nous semble important de signaler que nous voulons saisir la négation par une stratégie « bottom-up » :

15 1. Le point de départ de notre analyse est le simple repérage morphologique (donc aucunément sémantique) des formes traditionnelles de la négation, telles qu'elles sont décrites dans des ouvrages como l'Esbozo de la Gramática Española de la Real Academia Española (1973, 354-356) ou même le Diccionario de María Moliner, et qui reviennent aux formes suivantes : 


\begin{tabular}{|l|l|}
\hline$*$ adverbes & no, tampoco, nunca, jamás \\
\hline * pronoms & nada, nadie, ninguno \\
\hline$*$ adjectif & ninguno \\
\hline * prépositions & in \\
\hline * conjonction & ni \\
\hline * préfixes & a-, des-, in- \\
\hline
\end{tabular}

Cependant, la lecture de María Moliner, qui commente les différentes négations sous l'entrée "negar ", nous incite à ajouter à notre liste les formes dérivées de ce verbe «negar» (negación, negativo, negativamente, etc.), qu'on peut considérer comme des négations métalinguistiques, mais dont la forme est toujours facile à reconnaître.

Une lecture attentive de notre article nous permet de comptabiliser 29 négations présentes de formes explicite. Cela n'est pas peu pour un texte aussi court. Malgré le fait que l'adverbe no fournit presque un tiers des formes présentes - ce qui est peu surprenant - nous constatons la présence de la plupart des négations fondamentales: ni, nada, nunca, nadie, sin, a-, des-, in-, ninguno, ainsi que la négation métalinguistique negativa.

2. Après notre lecture strictement axée sur la morphologie, nous passons au niveau de la phrase, c'est-à-dire, de la syntaxe, qu'on peut considérer - à l'exception des cas où il s'agit de négations préfixales - comme dimension minimale pour l'étude du fonctionnement de la négation. Dû au fait que nous travaillons sur un corpus qui nous fournit des phrases « ad random», en d'autres mots, en absence des conditions «idéales» offertes par la consultation de notre propre "compétence ", il s'avère assez vite qu'il n'y a pas de relation $1: 1$ entre chaque élément négatif et la phrase dans laquelle celui-ci se trouve. Nous voyons qu'il se produit assez souvent des conglomérations de négations, raison pour laquelle nous n'avons pas seulement souligné les négations dans notre texte, mais nous avons aussi numeroté ces différentes conglomérations.

19 3. Cependant, nous sommes d'avis que l'analyse sémantique de la négation ne peut pas se limiter au niveau de la phrase ou de la syntaxe. Nous croyons, au contraire, qu'en reliant entre elles les différentes négations, nous pouvons découvrir quelle est leur fonction structurante à un niveau plus global. Cela ne peut être étudié à l'intérieur d'un texte.

\section{Qu'est-ce que nous voulons dire par « texte »?}

20 Nous savons que dans la langue quotidienne la notion « texte » renvoie tout d'abord par métonymie à la présence matérielle - sur le papier, sur un écran - de signes appartenant à un code linguistique - ; à un niveau plus strictement linguistique, «texte " renvoie au produit concret d'un code, c'est-à dire, à une langue déterminée, présente à travers son lexique, sa grammaire et se déroulant de façon linéaire.

21 Mais c'est surtout la définition théorique de "texte » telle qu'elle est donnée par la linguïstique discursive, et plus concrètement, par van Dijk, comme « séquences d'énoncés 
qui possèdent une cohérence globale, grâce à une macrostructure » $(1989,55)$ qui nous intéresse, par le fait qu'elle dépasse la linéarité des phrases juxtaposées et transforme le texte en architecture. Dans Cuba: una Numancia en el Caribe, ce sont le titre principal (Cuba : una Numancia en el Caribe) et les titres intercalés (Rendición sin condiciones Sacrificio lamentable) qui sont les indices les plus clairs de telles « macrostructures ». Cependant, il nous semble que nous ne pouvons pas ignorer que van Dijk parle également de deux autres structures, l'une supérieure - la superstructure - et l'autre inférieure - la microstructure - qui, dans le cas de notre article seraient présentes de la façon suivante : 1. La superstructure ou forme textuelle $(1989,142)$ se situe à un niveau qu'on peut considérer comme supérieur à celui de la macrostructure - dont elle est assez indépendante, d'ailleurs - et serait le reflet du "genre » auquel appartient le texte en question. Cuba: una Numancia en el Caribe, quant à sa superstructure, est un produit hybride, puisqu'il réunit le double titre et le chapeau - typiques de l'article informatif - et le caractère pyramidal de l'article argumentatif. Cette forme hybride, comme nous verrons, n'affecte pas la macrostructure comme telle, mais contribue certainement à son efficacité.

2. La microstructure qui, d'après van Dijk, n'est pas toujours facile à distinguer de la macrostructure $(1989,56)$, offre les éléments qui, par exemple, au moyen de la procédure de généralisation, fournissent la macrostructure. Dans notre texte, nous pouvons dire que chaque négation ou conglomération de négations offre une microstructure.

3. Il nous semble assez important de faire usage également de la notion de «structure rhétorique », conçue par van Dijk $(1989,126)$ comme une structure qui, à l'aide de certains éléments grammaticaux, contribue non à l'esthétique mais bien à l'efficacité du texte. Parmi les «figures» rhétoriques, dont van Dijk dit qu'elles interviennent dans les structures rhétoriques en question, celles qui nous intéressent le plus sont les "metasémèmes/métalogismes para suppression/adjonction négative (oxymore, ironie, paradoxe, litote) telle que les définit le Groupe $\mu(1982,49)$, et qui, très souvent, se formulent à l'aide d'une négation. Indépendemment du fait qu'elles pourraient produire un effet esthétique, le plus important est de savoir si ces figures ne dessineraient pas, comme le suggère van Dijk (1989 :126), la (ou les) macrostructure(s) du texte.

Dans Cuba: una Numancia en el Caribe, nous avons pu constater la présence d'une macrostructure à trois niveaux, à trois figures - antithèse(s) analogie, paradoxe -, dont la construction est largement dépendante des négations présentes. Cependant, en regardant dans un mouvement «top-down » depuis le niveau de la macrostructure vers le niveau sous-jacent des microstructures, nous voyons qu'en plus des négations repérées depuis le premier moment, de nombreux éléments non identifiables de façon morphologique comme des négations, rentrent dans les mêmes structures par d'autres moyens. Nous nous occuperons à peine de ces derniers : une étude plus poussée que la présente devra en dresser l'inventaire.

\section{Analyse des macrostructures superposées}

\section{Première macrostructure}

Dans les paragraphes $\$ 1$ à $\$ 7$ nous voyons comment par les moyens les plus divers, mais le plus souvent par des structures syntaxiques comprenant des négations, s'installent toute 
une série d'antithèses dans le texte. Même si ces antithèses n'ont pas toujours la même force - parfois elle sont symétriques et explicites, parfois elles sont asymétriques et jouent sur des présupposés - nous constatons qu'elles contribuent en une très large mesure à ce que Mortara Garavelli appelle une « intensification rhétorique» $(1988,277)$. L'antithèse dominante est, bien sûr, celle qui oppose Numancia aux Romains; mais d'autres antithèses jouant sur les contradictions internes de l'un de deux termes, ou hyperbolisant soit Numancia soit l'adversaire romain peuvent la renforcer. Une vision «top-down » nous permet de conclure que les deux titres intercalés explicitent cette opposition entre Numancia et les Romains.

\section{a. antithèses entre deux sujets différents}

* Grecs/Romains : dans la microstructure $\mathrm{n}^{\circ} 1$ la présence de inculto, précédé par son antonyme culto attire notre attention sur une antithèse sous forme de chiasme.

* Numancia/Romains : dans la microstructure $\mathrm{n}^{\circ} 3$, la négation lexicale desnaturalizada, à la fois en symétrie sémantique mais en antithèse formelle (par son préfixe négatif) avec materialista, a la fonction d'hyperboliser l'intransigeance de Scipion dans l'opposition Numancia/Rome, qui est explicitée plus loin dans la phrase.

* Romains1/Romains2 : la microstructure $n^{\circ} 4$ nous offre une antithèse construite au moyen d'une phrase relative qui oppose deux " générations " de romains en usant des synonymes dont le second est nié : cosechar/no pudieron alcanzar.

$31{ }^{*}$ Tribus/Romains : la microstructure $\mathrm{n}^{0} 6$ nous montre comment on peut préférer les formules passives fueron disuadidas et no ser ayudadas pour situer les sujet tribus face à l'adversaire Romains. C'est surtout l'expression litotique la amenaza de no ser ayudadas, qui nous semble intéressante, puisque elle ajoute une nouvelle antithèse - cette fois-ci, explicite - (ayudar/no ayudar) à amenaza, qui, déjà, définit les relations entre les deux.

$32{ }^{*}$ Numancia/Romains : dans la microstructure $n^{\circ}$ 7, l'énumération négative sin...no...ni...ni a la fonction d'hyperboliser l'attitude intransigeante de Scipion dans l'opposition Numancia/Romains.

$33 *$ Numancia/Romains : dans la microstructure $\mathrm{n}^{\mathrm{0}} 8$, l'énumération inaugurée par sin a la fonction d'hyperboliser la agonía, qui exprime l'opposition entre Numancia et les Romains.

$34{ }^{*}$ Numancia/Romains : la microstructure $\mathrm{n}^{\circ} 9$ fait usage également d'une subordonnée relative pour opposer ideales...que era inútil tratar de entender.

$35 *$ Numancia/Romains : dans la microstructure $\mathrm{n}^{\circ}$ 10, le mot independencia nous suggère par sa morphologie l'alternative dependencia/ independencia qui s'offre aux habitants de Numancia.

36 * Numancia/Romains: la microstructure $\mathrm{n}^{\circ} 11$ nous donne par sa négation métalinguistique la negativa une mention très claire de l'opposition entre Numancia et les Romains, cette fois-ci en nous décrivant un acte de parole qui en est l'expression.

\section{b. antithèses à l'intérieur du même sujet}

37 * Romains/Romains : dans la microstructure no 2 nous voyons que temidas....incapaces nous donne une antithèse symétrique ayant la forme d'un oxymore. 
Numancia/Numancia : la microstructure nº 8 Pero Numancia no se rindió, plutôt que de nous intéresser pour rindió, qui est une mention de la relation Numancia/Rome, nous semble intéressante par la présence de pero...no qui met en place une antithèse entre le présupposé (se rindió) et ce qui s'est produit réellement (no se rindió).

* Romains/Romains : la microstructure $n^{\circ} 12$ la fuerza y la brutalidad,no ..la razón nous montre une antithèse symétrique qui naît de la volonté de produire un effet de répétition au moyen de la négation de l'antonyme. Le but de ce jeu est moins l'effet de paraphrase que l'effet d'antithèse. Remarquons qu'à l'intérieur de la même microstructure, nous trouvons nadie ha discutido nunca que..., qui a très clairement la fonction de renforcer (hyperboliser) cette antithèse.

* Numancia/Numancia: la microstructure no 13 présente exactement les mêmes caractéristiques que le $n^{\circ} \mathbf{8}$, sauf qu'elle est accompagnée de l'expression négative $n i$ mucho menos, à fonction clairement hyperbolique.

$41{ }^{*}$ Quién/Todos : la microstructure $\mathrm{n}^{\circ} 14$ nous offre une antithèse qui a pris la forme d'interrogation rhétorique et qui, par le fait qu'elle n'oppose par Numancia/Romains, mais qu'elle est centrée sur Numancia, a la fonction d'hyperbole. En effet, le but de la formulation ¿Quien defendería que....no? est d'obtenir la réponse contraire Todos defenderían....que sí, qui renforcerait clairement la position de Numancia dans son conflit avec les Romains.

* Numancia/Numancia : la microstructure n 15 elegir su propio futuro,o ...no tener ninguno nous montre comment une antithèse peut naître de la présence de la conjonction $o$ entre deux verbes plus ou moins synonymes. L'adjectif ninguno joue, par sa fonction quantificatrice, le rôle d'hyperbole de no tener.

\section{Deuxième macrostructure}

Au-delà du niveau des différentes antithèses mises en place dans les paragraphes $\$ 1$ à $\$ 7$ et renforcées au niveau macrostructurel par les titres intercalés -, le paragraphe $\$ 8$ nous permet de passer à une macrostructure supérieure. En effet, comme le souligne van Dijk $(1989,56)$, rien n'empêche une construction hiérarchique de plusieurs macrostructures : dans notre texte, nous trouvons à ce niveau supérieur l'analogie annoncée par le titre Cuba: una Numancia en el Caribe. Perelman a décrit en détail (1970, § 82-88) comment le phore (ici : Numancia et ses phores parallèles) est mis en place en fonction du thème (ici : Cuba); ce qui, dans notre texte, veut dire que toutes les antithèses mises en place pour décrire les oppositions à l'intérieur du phore peuvent, en principe, être extrapolées vers le thème. C'est justement au niveau de cette deuxième macrostructure, mise en place par muchas similitudes, mismo cerco, aún más cobarde et annoncée depuis le titre, que nous comprenons que la quantité et la précision des antithèses de la macrostructure inférieure ne visait pas seulement une description détaillée de l'opposition entre Numancia et les Romains, mais prévoyait déjà la projection realisée au moyen de l'analogie. Le thème, qui est la nouvelle antithèse Cuba/Etats-Unis, apparaît de façon très claire, formulé également par une antithèse dans la microstructure No parece importar que...siempre que... qui, comme nous pouvons constater, comporte également une négation. 


\section{Troisième macrostructure}

Cependant, il faut être attentif et ne pas croire qu'avec la réapparition de l'analogie du titre dans le paragraphe $\$ 8$ tout est dit. Bien au contraire, nous constatons qu'à l'intérieur du paragraphe $\S 9$, le dernier du texte, s'installe une nouvelle macrostructure, absente du titre Cuba: una Numancia en el Caribe, mais présente dans le chapeau de l'article; macrostructure qui, comme il se doit, englobe les deux précédentes. Dans ce dernier paragraphe - d'un article qui par sa structure pyramidale nous réserve le climax pour la fin - nous observons ce que Perelman appelle le « développement » ou le «prolongement de l'analogie" $(1970, \S 85)$. Dans notre texte,il s'agit du mouvement par lequel se réalise la confrontation des quatre termes de l'analogie en suggérant le rapprochement des termes Numancia/Cuba. Le caractère inadéquat de cette analogie à trois termes est signalée immédiatement par la historia no enseña casi nada et desconocer qui donnent comme résultat impasible, analogue au no parece importar du paragraphe précédent. Le rejet de l'analogie permet la mise en place de la figure appelée paradoxe, présente dans le texte par l'expression ironía de la historia. Nous voyons donc que l'idée de départ du texte, l'idée qui l'a fait naître et qui lui donne sa cohérence ultime est la figure négative du paradoxe. Finalement, imperdonable, bien que faîte du texte, à notre avis, ne peut pas être consideré comme l'indice d'une autre macrostructure, supérieure à celle du paradoxe, mais nous semble, au contraire, inséparable de celle-ci.

\section{Conclusion}

Une lecture tout d'abord linéaire et ensuite tubulaire du réseau sémantique formé par les négations à présence explicite dans Cuba: una Numancia en el Caribe, nous a permis de voir comment un texte peut utiliser ses microstructures pour construire plusieurs macrostructures superposées, et comment la négation peut contribuer a une plus grande efficacité de la force persuasive de ces macrostructures.

$\mathrm{Au}$ niveau de la première macrostructure, nous constatons comment de nombreuses négations peuvent mettre en place une série d'antithèses renforcées par diverses hyperboles également négatives.

Au niveau de la deuxième macrostructure, nous constatons que l'analogie tire une grande efficacité de la clarté de antithèses mises en place à un niveau inférieur.

Au niveau de la troisième macrostructure, se réalise de façon surprenante le rejet de l'analogie entre les termes Espagne/Numancia, de façon que le texte se termine par une nouvelle " antithèse" - mais de niveau infiniment supérieur aux antithèses de la première macrostructure - du type "paradoxe", que le texte l'appelle íronía de la historia et que Alicia $\mathrm{M}^{\mathrm{a}}$ Canto considère imperdonable.

Il nous semble que notre texte fait un usage très efficace du moyen d'intensification rhétorique qu'est la négation: des moyens "négatifs" de toutes sortes convergent pour construire un raisonnement, sans que par les mouvements successifs - et surtout par le passage à une macrostructure supérieure - se produisent des ambiguïtés. C'est ce jeu très complexe de structuration, qui suggère une vraie architecture grâce aux figures rhétoriques macrostructurellement superposées - et sans doute observable dans d'autres textes à caractère argumentatif -, que nous appelons la rhétorique de la négation. 


\section{BIBLIOGRAPHIE}

BOSQUE, Ignacio, 1980, Sobre la negación, Madrid, Cátedra.

GROUPE $\mu, 1982$, Rhétorique générale, Paris, Seuil.

IBAÑEZ, Roberto, 1972, Negation im Spanischen, München, Fink Verlag.

MOLINER, María, 1973, Diccionario de uso de la lengua española, Madrid, Gredos.

MORTARA GARAVELLI, Bice,

1991, Manual de retórica, Madrid, Cátedra.

PERELMAN,Ch.,OLBRECHTS-TYTECA,L,

1970, Traité de l'argumentation. La nouvelle rhétorique,Bruxelles, Ed. de l'Université de Bruxelles.

REAL ACADEMIA ESPAÑOLA,

1973, Esbozo de una nueva gramática de la lengua española, Madrid, Espasa-Calpe.

VAN DIJK,Teun, 1989, La ciencia del texto. Un enfoque interdisciplinario, Barcelona, Paidos.

VOIGT, Burkhard, 1979, Die Negation in der spanischen Gegenwartsprache. Analyse einer linguistischen Kategorie, Frankfurt am Main, Peter Lang.

\section{RÉSUMÉS}

Afin d'explorer les possibilités d'une approche transphrastique de la négation, nous avons choisi comme corpus un texte journaliste du type argumentatif et comme principe théorique la distinction faite par Teun van Dijk entre micro, macro et superstructures sémantiques, ainsi que structures rhétoriques. A travers notre analyse, nous constaterons que les différentes négations morphologiquement repérables, loin d'être des éléments fortuits, construisent par leur pouvoir créateur d'antithèses une vraie structure rhétorique garantissant au texte sa cohérence, sa clarté et son efficacité argumentative.

In order to check to what result leads a "transsentential" study of negation, we chose a newspaper article of argumentative type, and we took up the theorical distinction assumed by Teun van Dijk between semantical micro-, macro- and superstructures, as well as rhetorical structures. We discover through this analysis that the various morphological markers do not occur accidentally, but that they manage, by creating antitheses, to build up a real rhetorical structure that guarantees the text's coherence, its clarity and its argumentative efficiency.

\section{AUTEUR}

\section{HILDEGARD VERMEIREN}

PHVT - Ecole d'Interprètes de Gand-Belgique 